\title{
TOPOLOGICAL EVOLUTION AND COSMIC CHAOS
}

\author{
ROMAN TOMASCHITZ \\ Inter-University Centre for Astronomy and Astrophysics, Post Bag 4, Ganeshkhind, Pune, 411 007, India \\ and Indian Institute of Astrophysics, Koramangala, Bangalore 560 034, India
}

(Received January 17, 1997)

\begin{abstract}
An account on the origins of cosmic chaos and its physical impact in an open and multiply connected universe is given. A new type of cosmic evolution by topological deformations, unpredicted by Einstein's equations, is pointed out. The chaoticity of the galactic world-lines provides a mechanism to create the galactic equidistribution. Global metrical deformations of the open and multiply connected spacelike slices induce angular fluctuations in the temperature of the microwave background. They cause backscattering of electromagnetic fields and particle creation in quantum fields. The topological microstructure of spacetime provides a mechanism for $\mathrm{CP}$ violation by self-interference.
\end{abstract}

\section{Introduction}

Our contemporary understanding of the global structure of the Universe is based on the assumption of a spacetime continuum, a curved four-dimensional world. The observational evidence for this is actually scarce, but it offers at any rate a very simple qualitative explanation of the cosmic red-shifts. The second motivation to model the Universe as a Riemannian four-manifold is the successful application of Riemannian geometry in explaining planetary perihelion shifts, and the deflection of light by gravitating bodies. These are local phenomena, which can be explained by introducing a Riemannian metric on a single co-ordinate chart. In cosmology, however, we are concerned with the global structure, with the topology of the four-manifold [1-3].

In local problems of general relativity, on a single co-ordinate chart, Einstein's equations determine completely the evolution of the metric, once the energy-momentum tensor of the local gravitational sources is known. In cosmology we have to consider the evolution of the metric as well as the evolution of the topology of spacetime. Einstein's equations certainly do not give any hint on the topology, and the evolution of the metric is likewise undetermined, because we do not really know the energy-momentum tensor of the matter distribution in the Universe, nor the boundary conditions to be imposed. It is, in my opinion, pointless to hunt for elusive laws of cosmic evolution, the modeling employed in such endeavours is always copied from finite classical or quantum systems, and there is no reason to assume that the Universe is a finite Hamiltonian system like any other. A much more promising 
approach to cosmology is to figure out possibilities of cosmic evolution, and to think over which local physical manifestations they can have.

The actual question is not so much what is the topology, but rather how does it evolve, because otherwise it is difficult to motivate why the 3-space should have acquired, once and for all, a particular type of topology and metric. What we will advocate here is a cosmology with an open 3-space that evolves by global metrical deformations, which can give rise to transitions from one topology to another [4-7]. We do not know the present topology of the Universe, and so we have to content ourselves to figure out possible physical effects of a multiply connected topology. In Section 3 we will review some local manifestations of the global 3-space topology, but at first I outline a cosmic evolution which goes far beyond the usual scaling of the length unit in traditional Robertson-Walker (RW) cosmology.

\section{Topological evolution by global metrical deformations of the 3-space}

In [4-7] a cosmology was introduced in which the 3-space is negatively curved, open, and topologically multiply connected. These three conditions allow topological evolution by global deformations. The negatively curved spacelike slices must be open and multiply connected, otherwise they cannot be deformed without destroying the constant curvature. The negative curvature (local hyperbolicity) is necessary to make the world-lines unstable, and the multiple connectivity can trap them and confine to a finite region, the chaotic nucleus of the open 3-space. This confinement, and the local hyperbolicity renders world-lines chaotic (Bernoulli property). The size of the nucleus depends on the expansion factor and, above all, on the topology of the 3-slices.

Chaoticity is an efficient mechanism to create an equidistribution, but the actual problem is to explain the inhomogeneities in the galactic distribution. The time evolution of the world-lines depends on the expansion and on the global deformations that the 3-space undergoes. Concepts like mixing and ergodicity, which are commonly used in Hamiltonian dynamics to describe the degree of chaoticity [8,9], do not reflect the time evolution of the system. They are based on the geometric shapes of

Figure 1. The horizon at infinity of the Poincare half-space $H^{3}$, the universal covering space of the spacelike 3-sections of the RW cosmology. A spacelike slice $(F, \Gamma)$ is realized in $H^{3}$ as a polyhedron $F$ with face-identification. The face-pairing transformations generate a discrete group $\Gamma$ which gives, if applied to the polyhedron, a tessellation $\Gamma(F)$ of $H^{3}$ with polyhedral images. This tessellation induces by continuity also a tiling on the boundary of $H^{3}$, which is depicted here. The complete tiling of $H^{3}$ is obtained by placing hemispheres onto the circular arcs. This polyhedral tiling of hyperbolic space $H^{3}$ is the covering space construction for the RW geometry.

The qualitative structure of the fractal limit set $\Lambda(\Gamma)$ depends on the topology of the 3-slices, which is in turn determined by the covering group [20, 21]. In this example the 3-space fibers over an open interval, with Riemann surfaces $(g=49)$ as fibers.

The chaotic trajectories have covering trajectories with initial and terminal points in the limit set. If the end points are outside $\Lambda(\Gamma)$, but close to it, then the trajectory is regular, but it is shadowed by trapped chaotic trajectories over a long period before leaving the chaotic region. The tiling on the boundary of $H^{3}$ is the key to determine this chaotic nucleus of the open 3 -space $[5,11]$. 


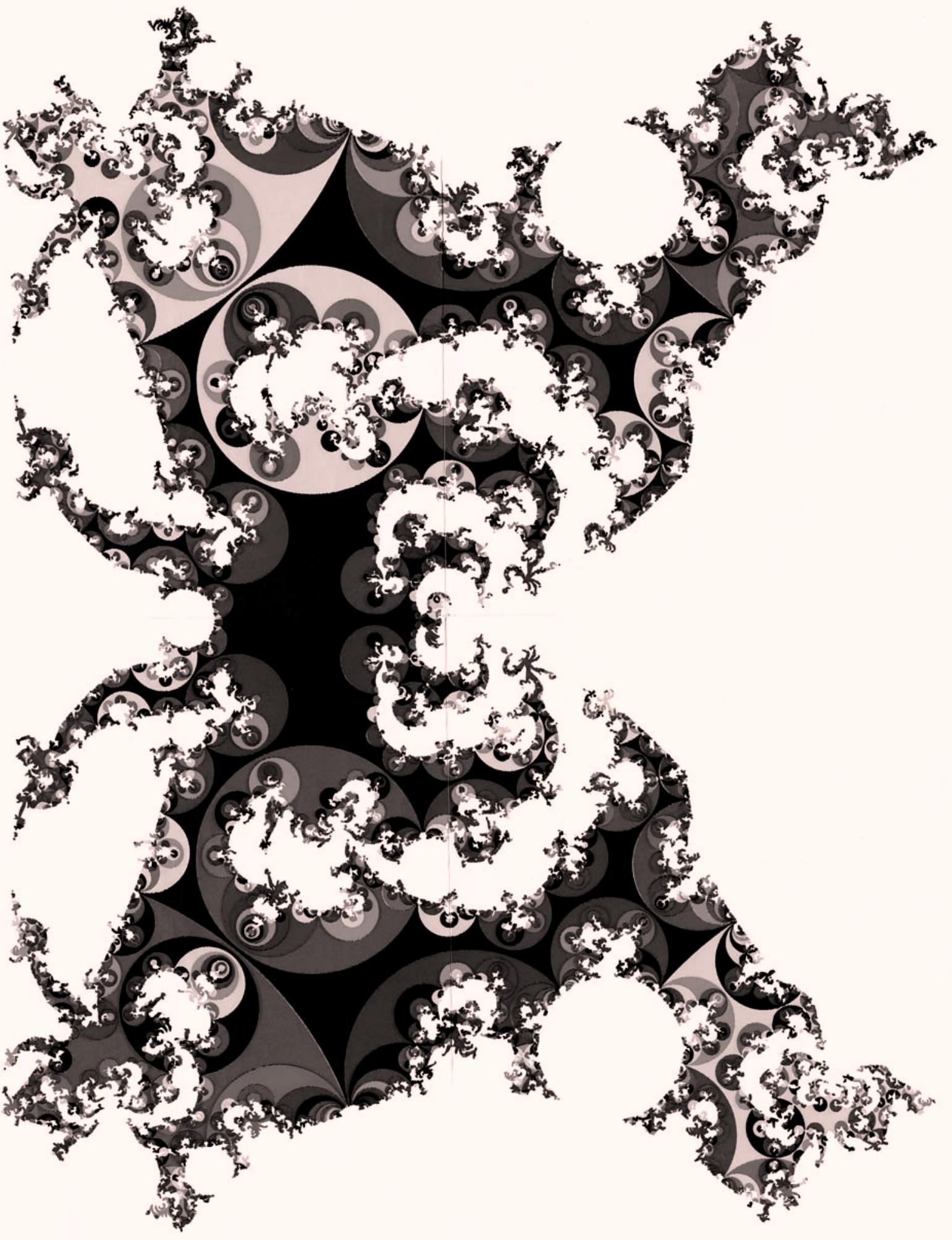


trajectories of infinite length. Here the question is what happens in finite times, and with world-lines of finite length [4, 7]. Regular trajectories entering the chaotic center of the 3-space are shadowed by chaotic ones, before they ultimately diffuse out of the center into the open 3-space. The mixing in the center tends to create a uniform distribution, but inhomogeneities will always remain in a finite time [10].

The center of the multiply connected 3-space is geometrically determined by the convex hull of the limit set of the covering group, its explicit construction is a matter of elementary geometry. Regular trajectories are shadowed by chaotic ones whenever their covering geodesics have initial or terminal points close to the limit set, cf. Fig. 1.

The multiple connectivity of the open 3-space provides a new type of cosmic evolution by global metrical deformations. 'Global' means here a deformation which does not locally change the curvature of the 3-space. Two spacelike sections which have the same topology and constant curvature (apart from a trivial rescaling by the expansion factor) may be globally, as 3-manifolds, nonisometric. If the 3-space is open and multiply connected, it can be endowed with a variety of metrics, all giving rise to the same constant curvature. These metrics are labeled by a finite number of independent parameters, the dimension of this parameter space depends on the topology of the spacelike slices. The line element of the extended RW cosmology is $d s^{2}=-c^{2} d t^{2}+a^{2}(t) g_{i j}(y(t), \mathbf{x}) d x^{i} d x^{j}$. Here $g_{i j}\left(y_{1}, \ldots, y_{n}, \mathbf{x}\right)$ denotes a continuous family of hyperbolic metrics of constant negative curvature -1 . The parameters $y_{k}$ may be chosen to vary in a compact domain of $\mathbb{R}^{n}$. The evolution of the 3-space is characterized by a time-dependent path $y(t)$ in this deformation space, and by the expansion factor $a(t)$. If the path reaches the boundary of the deformation space, a topology change occurs, the connectivity of the 3-space can change or it may even disintegrate, leaving behind cusp singularities on its newly emerging components. The curvature of the 3-space stays constant (apart of the mentioned rescalling) during the whole deformation process. For an exactly solvable example of a global deformation see $[11,6]$.

Remark: In [12] we considered local distortions of the constantly curved 3-space geometry by gravitational waves. Constant curvature is not really necessary for the evolution mechanism described here, it just ensures that this topological evolution does not occur at the expense of creating large local inhomogeneities in the curvature. If the 3-space metric is uniformly close to a metric of constant negative curvature, it will stay so during a global deformation.

The topology, the dynamics induced by it via global deformations, and the resulting topology changes are not predictable by Einstein's equations, which nevertheless impose restrictions on the speed and size of global deformations, because the energy and pressure densities defined by the Einstein tensor must stay positive.

\section{Physical manifestations of the topological 3-space structure}

Objections against open cosmologies are sometimes based on the argument that we will not be able to look at infinity and to verify what is happening there. What is overlooked here is that the topological structure of the 3-space can manifest it- 
self locally. We will give now some examples. I also mention here that an entirely different approach to the problem of "how to look at infinity" in an open universe was suggested in [10], where tachyonic (i.e. superluminal [13, 14]) signal transfer is considered in the distinguished Machian frame of reference defined by the galactic grid.

\section{Angular fluctuations in the temperature of the microwave background radiation [15, 16]}

If one studies the impact of global deformations of the open 3-space on the free photon gas [4], one has to take two time scales into account: cosmic time as defined by the expansion factor, and the time scale on which the deformations take place. The latter is determined by the time parametrization of the path $y(t)$, which determines the metric on the spacelike sections. Only if the scale on which noticeable variations of $g_{i j}(y(t), \mathbf{x})$ occur is much larger than the time scale determined by the expansion factor, one has a really uniform expansion.

In the following we assume that $g_{i j}(y(t), \mathbf{x})$ adiabatically varies on the time scale of the cosmic expansion. We use the universal covering space construction as sketched in the Caption of Fig. 1. A global metrical deformation of the 3-space is realized by deforming the Poincaré metric $\widetilde{g}_{i j}$ of the covering space $H^{3}$, cf. [4] for details. We consider a small adiabatic deformation of this metric, $g_{i j}(y(t), \mathbf{x})=\widetilde{g}_{i j}(\mathbf{x})+h_{i j}(t, \mathbf{x}), h_{i j}$ is a symmetric tensor field periodic with respect to the covering group $\Gamma$, i.e., $h_{i j}(t, \mathbf{x})=$ $h_{m n}(t, \gamma(\mathbf{x}))\left[\gamma^{\prime} \mathbf{x}\right]_{i}^{m}\left[\gamma^{\prime} \mathbf{x}\right]_{j}^{n}$, for all $\gamma \in \Gamma ;\left[\gamma^{\prime} \mathbf{x}\right]$ denotes the Jacobian of the transformation $\gamma$. The idea is simply to calculate, semiclassically, via the eikonal equation $g^{\mathrm{RW} \mu \nu} \psi_{, \mu} \psi_{, \nu}=0$ (with the extended RW metric $g_{00}^{\mathrm{RW}}=-c^{2}, g_{i j}^{\mathrm{RW}}=a^{2}(t) g_{i j}(y(t), \mathbf{x})$, $g_{0 j}^{\mathrm{RW}}=0$ ), frequency shifts induced by the deformation $h_{i j}(t, \mathbf{x})$. The perturbed eikonal for rays issuing at a point $\eta$ at infinity of the covering space $H^{3}$ is $\psi=\widetilde{\psi}+\chi(t, \mathbf{x}, \eta)$, with $\widetilde{\psi}$ a solution of $\tilde{g}^{\mathrm{RW} \mu \nu} \widetilde{\psi}_{, \mu} \widetilde{\psi}_{, \nu}=0$. Here $\widetilde{g}_{\mu \nu}^{\mathrm{RW}}$ is defined, like $g_{\mu \nu}^{\mathrm{RW}}$, with $g_{i j}$ replaced by $\tilde{g}_{i j}$. The frequencies are shifted by $\nu \longrightarrow \nu+\frac{1}{2 \pi} \partial \chi / \partial t$. In the Planck distribution, $\rho d \nu=8 \pi h c^{-3} \nu^{3}(\exp (h \nu / k T)-1)^{-1} d \nu$, these shifts can be absorbed by introducing a new temperature variable $\widetilde{T} \approx T\left(1-\frac{1}{2 \pi} \nu^{-1} \partial \chi(t, \mathbf{x}, \eta) / \partial t\right)$. This amounts to a weak angular dependence $\eta$ of the temperature.

Particle creation in quantum fields and backscattering of electromagnetic radiation

In simply connected Robertson-Walker cosmologies the variation of the expansion factor leads to particle creation. However, this cannot happen in neutrino or electromagnetic fields, because in the solutions of the corresponding wave equations the expansion factor scales out with a simple power law. But global metrical deformations of the 3-space do create particles and backscattering even in conformally coupled fields $[4,6]$. The point here is that during a deformation the wave equation is not time separable, and a wave initially composed of positive frequencies will acquire negative frequency modes. So, antiparticles emerge in quantum fields, and backscattered wave trains in classical fields. 


\section{Topological CP violation by self-interference}

The reasons for $\mathrm{CP}$ violation are as yet not really understood. This is so despite, or perhaps because, this extraordinary phenomenon could be easily incorporated into particle physics by adding symmetry breaking interactions to the Lagrangians. The space-reflection symmetry is of course always present in classical mechanics, its violation is a pure quantum effect [17]. In [4] it was therefore suggested to explain parity violation as a topological interference phenomenon, by adopting Weyl's idea to associate elementary particles with the microstructure of the 3-space [18, 19]. A space-reflected wave can wrap around a microscopic geodesic loop, and this causes self-interference [11], which destroys the antiunitarity of the parity operator. Thus parity is a broken symmetry already on the level of free wave equations.

Some open research problems in extended RW cosmology are outlined in [10]. In [22] perihelion shifts are explained in terms of a scalar gravitational field and a permeability tensor representing the physical substance of space-the ether.

I have tried to show here that the topology and the topological dynamics of an open universe may have very concrete physical manifestations, and that the topology of spacetime is a dynamical object, able to evolve like the metric in local problems of general relativity.

\section{Acknowledgments}

The author acknowledges the support of the Japan Society for the Promotion of Science, contract no. P-95378. The hospitality and stimulating atmosphere of the Institute of Mathematical Sciences, Madras, and the Centre for Nonlinear Dynamics, Bharathidasan University, Trichy, are likewise gratefully acknowledged.

\section{REFERENCES}

[1] L. Infeld and A. Schild: Phys. Rev. 68 (1945), 250.

[2] E. Schrödinger: Expanding Universes, Cambridge University Press, Cambridge 1956.

[3] O. Heckmann and E. Schücking: Handbuch der Physik 53 (1959), 489.

[4] R. Tomaschitz: J. Math. Phys. 32 (1991), 2571; ibid. 34 (1993), 1022; ibid. 34 (1993), 3133; ibid. 35 (1994), 1573.

[5] R. Tomaschitz: Complex Systems 6 (1992), 137.

[6] R. Tomaschitz: in Deterministic Chaos in General Relativity, eds. D. Hobill et al., Plenum, New York 1994, p. 251.

[7] R. Tomaschitz: in Chaotic Dynamics: Theory and Practice, ed. T. Bountis, Plenum, New York 1992, p. 161.

[8] V. I. Arnold: Mathematical Methods of Classical Mechanics, Springer, New York 1978.

[9] M. C. Gutzwiller: Chaos in Classical and Quantum Mechanics, Springer, New York 1990.

[10] R. Tomaschitz: Chaos, Solitons and Fractals 7 (1996), 753; ibid. 8 (1997), 761.

[11] R. Tomaschitz: Int. J. Theor. Phys. 33 (1994), 353.

[12] R. Tomaschitz: Int. J. Theor. Phys. 36 (1997), 955.

[13] O. Bilaniuk, V. Deshpande and E. Sudarshan: Am. J. Phys. 30 (1962), 718.

[14] G. Feinberg: Phys. Rev. 159 (1967), 1089.

[15] R. K. Sachs and A.M. Wolfe: Astrophys. J. 147 (1967), 73.

[16] G. F. Smoot et al.: Astrophys. J. 396 (1992), L1.

[17] C.N. Yang: in The Physicist's Conception of Nature, ed. J. Mehra, D. Reidel, Dordrecht 1973. 
[18] H. Weyl: Space-Time-Matter, Dover Publ., New York 1951.

[19] M.S. El Naschie: in Quantum Mechanics, Diffusion, and Chaotic Fractals, eds. M.S. El Naschie et al., Elsevier, Oxford 1995, p. 93.

[20] R. Fricke and F. Klein: Forlesungen über die Theorie der automorphen Funktionen, vols. 1, 2, Johnson Reprint, New York 1965.

[21] F. Klein and R. Fricke: Vorlesungen über die Theorie der elliptischen Modulfunktionen, vols. 1, 2, Johnson Reprint, New York 1965.

[22] R. Tomaschitz: Chaos, Solitions and Fractals 8 (1997), in press. 